\title{
Ewing Sarcoma in a Patient With Cowden Syndrome
}

\author{
Mod C. Chandhanayingyong, MDa,b; Nicholas M. Bernthal, MDa; Piti Ungarreevittaya, MD, ;
} Scott D. Nelson, MDc; Sant P. Chawla, MDe; and Arun S. Singh, MD

\begin{abstract}
A 47-year-old woman, initially diagnosed in 1996 with Cowden syndrome (CS), PTEN-mutant bilateral breast cancer, a thyroid nodule, and uterine fibroids, presented to UCLA in 2013 with Ewing sarcoma of the pelvic bone. Her treatment course included mastectomies, hysterectomy/oophorectomy, and total thyroid resection, and chemotherapy, radiation, and hemipelvectomy for Ewing sarcoma. This case report illustrates the unusual presentation of Ewing sarcoma in a patient with PTEN-mutant CS, the probable underlying molecular pathogenesis, long-term management, and therapeutic considerations. (J Natl Compr Canc Netw 2015;13:1310-1314)
\end{abstract}

\section{Case Summary}

A 47-year-old woman was originally diagnosed with breast cancer at age 29 years in 1996. She had a 7-mm well-differentiated infiltrating ductal carcinoma of her right breast with 3 of 15 lymph nodes positive. The tumor was estrogen receptor (ER)-positive and HER2/ neu-negative. She also had ductal carcinoma in situ (DCIS) in her contralateral left breast. After bilateral

From the aDepartment of Orthopaedic Surgery, David Geffen School of Medicine, UCLA, Los Angeles, California; ${ }^{b}$ Department of Orthopaedic Surgery, Faculty of Medicine Siriraj Hospital, Mahidol University, Bangkok, Thailand; 'Department of Pathology, UCLA, Los Angeles, California; dDepartment of Pathology, Khon Kaen University, Khon Kaen, Thailand; eSarcoma Oncology Center, Santa Monica, Santa Monica, California; and 'Department of Medicine,

Division of Hematology/Oncology, David Geffen School of Medicine, UCLA, Los Angeles, California.

Submitted February 27, 2015; accepted for publication July 16, 2015.

Dr. Chawla has disclosed that he receives research support from and is a consultant for CytRx, Amgen, Bayer, and Threshold. The remaining authors have disclosed that they have no financial interests, arrangements, affiliations, or commercial interests with the manufacturers of any products discussed in this article or their competitors.

Correspondence: Arun S. Singh, MD, UCLA, Department of Medicine, Division of Hematology/Oncology, 2020 Santa Monica Boulevard, Suite 600, Santa Monica, CA 90404.

E-mail: asingh@mednet.ucla.edu mastectomies, she received adjuvant chemotherapy with doxorubicin and cyclophosphamide (AC) followed by paclitaxel. She received 4 cycles of doxorubicin at $60 \mathrm{mg} / \mathrm{m}^{2}$. She was not given adjuvant tamoxifen because data supporting its adjuvant use was not mature at that time. In the setting of bilateral breast cancer in a young woman with maternal Ashkenazi Jewish ancestry, genetic testing for BRCA1/2 was recommended, which revealed her to be BRCA-negative. Given the history of uterine fibroids, thyroid nodules, breast cancer, and macrocephaly, a diagnosis of Cowden syndrome (CS) was considered and subsequently confirmed with the R130Q mutation in her PTEN gene. In 2008, she had a complete hysterectomy with salpingo-oophorectomy for uterine fibroids, and in 2009 she had a complete thyroidectomy for multicystic adenomatous nodules with focal papillary hyperplasia.

The patient was doing well until she developed rightsided hip pain in July 2012 that was thought to be exerciserelated. In October 2013, she had a fall that exacerbated this hip pain. Radiographic findings showed a fracture of her iliac wing through an expansile lytic lesion (Figure 1A). MRI showed a $4.1 \times 3.1 \times 5.6-\mathrm{cm}$ expansile lesion involving the iliac wing with adjacent soft tissue extension and a pathologic fracture (Figure 1B). PET/CT showed a localized pelvic lesion (Figure 1C). Biopsy results revealed a small blue round cell tumor (Figure 1D) positive for CD99 but negative for pancytokeratin, S100, Melan-A, desmin, myeloperoxidase, CD43, CD45, CD30, CD138, Kappa, Lambda, CK7, mammaglobin, ER, progesterone receptor, HER2/neu, and GCDFP-15. This specimen was sent for fluorescence in situ hybridization analysis and showed an EWSR1 gene rearrangement. Based on these results, the diagnosis of Ewing sarcoma was made. 


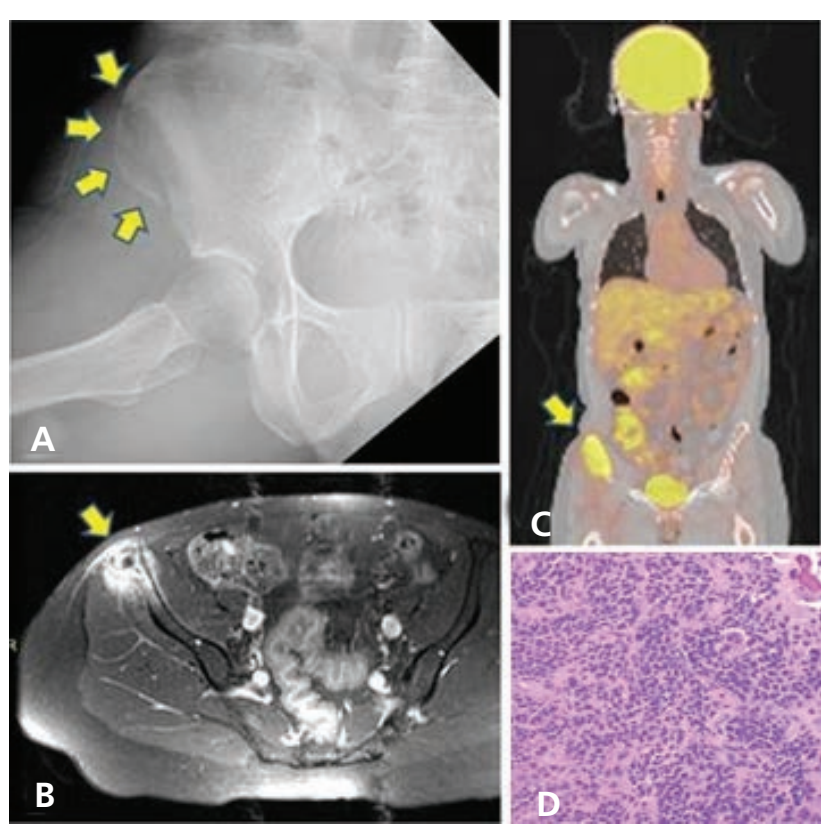

Figure 1 (A) An anteroposterior plain radiograph of the right pelvis demonstrates osteolytic lesion and pathologic fracture of the right anterior superior iliac spine (arrows). (B) An axial, fat-saturated T2 MRI shows a hyper-signal intensified $41 \times 31 \times 56-\mathrm{mm}$ mass within the right iliac wing and surrounding soft tissues (arrow). (C) A coronal FDG-PET/ CT scan shows high metabolic activity of the mass involving the right iliac bone with soft tissue component (arrow). (D) Sections from the biopsy of the right anterior iliac crest showed sheets and nests of relatively uniform small blue round cells in a fibrous background among bits of woven bone. The tumor cells displayed hyperchromatic nuclear chromatin and a small amount cytoplasm. Mitotic activity was increased and large areas of necrotic tumor were present. Immunohistochemistry studies showed the tumor cells to be positive for CD99 and negative for other markers, including pancytokeratin, Melan-A, myeloperoxidase, desmin, CD43, CD45, CD30, CD138, Kappa, Lambda, CK7, mammaglobin, estrogen receptor, progesterone receptor, HER2/neu, and GCDFP-15. Additionally, fluorescence in situ hybridization studies were positive for EWSR1 gene rearrangement.

Physical examination showed a well-appearing, highly functional woman without any noticeable impairment. She was noted to have macrocephaly, with an occipitofrontal circumference of $59.5 \mathrm{~cm}$ ( $>97$ th percentile). Surgical scars were found on her breasts, neck, and abdomen. Neurologic examination was intact, with no cerebellar signs or ataxia. There was no kyphoscoliosis. Skin and mucocutaneous examination showed facial papules, trichilemmomas (which were clinically diagnosed and not biopsy-confirmed) (Figure 2A), oral mucosal papillomas, and acral keratoses (Figure 2B,C). She had an ill-defined 3-cm mass at the right anterior superior iliac spine that was firm, fixed, and mildly tender. No cervical, axillary, or inguinal lymphadenopathy was noted. The patient's case was discussed at a multidisciplinary sarcoma tumor board and she was treated with 3 cycles of neoadjuvant chemotherapy consisting of vincristine, doxorubicin, and cyclophosphamide, alternat- ing with ifosfamide and etoposide. She also received 45 Gy of neoadjuvant radiation therapy to her right pelvis. Subsequently, she underwent a right hemipelvectomy of the superior iliac crest. On histology, her tumor was shown to have greater than $99 \%$ tumor necrosis with negative surgical margins. Her postoperative course was uneventful. She received adjuvant chemotherapy with the standard Ewing regimen. Interestingly, she reported decrement of her facial papules and trichilemmomas after chemotherapy.

\section{Discussion}

CS is a disorder that belongs in the group of PTEN hamartoma tumor syndromes (PHTs), which are characterized by germline mutations, mostly in the PTEN tumor suppressor gene, that carry an increased risk of certain types of tumors., ${ }^{1,2}$ Characteristic features of CS include multiple hamartomas presenting as benign skin tumors, such as multiple trichilemmomas, papillomatous papules, and acral keratosis. ${ }^{3,4}$ Other findings include intestinal hamartomatous polyps and dysplastic gangliocytomas of the cerebellum (Lhermitte-Duclos disease). ${ }^{5} \mathrm{~A}$ wide variation of skeletal abnormalities have been mentioned, but not proven to be associated with CS, including kyphoscoliosis, pectus excavatum, syndactyly, and maxillary, mandibular, and scapular hypoplasia. ${ }^{6}$

In this case, the patient initially presented with bilateral breast cancers, including a well-differentiated infiltrating ductal carcinoma on her right breast and DCIS on her left breast, at a young age. The diagnosis of CS was established based on the NCCN Clinical Practice Guidelines in Oncology (NCCN Guidelines) for Genetic/Familial High-Risk Assessment: Breast and Ovarian (to view the most recent version of these guidelines, visit NCCN.org). ${ }^{5}$ She had 3 major criteria: (1) breast cancers (bilateral), (2) macrocephaly, and (3) multiple mucocutaneous lesions, including acral keratoses ( $\geq 3$ acral hyperkeratotic papules) and facial trichilemmomas $(\geq 3$, clinical diagnosis). And she had one minor criterion: multicystic adenomatous nodules with focal papillary hyperplasia. Genetic sequencing confirmed an R130Q missense mutation in the PTEN gene.

$\mathrm{CS}$ is associated with an increased risk of developing breast, thyroid, endometrial, and renal cell cancers, with a lifetime risk as high as $85 \%, 35 \%, 28 \%$, 

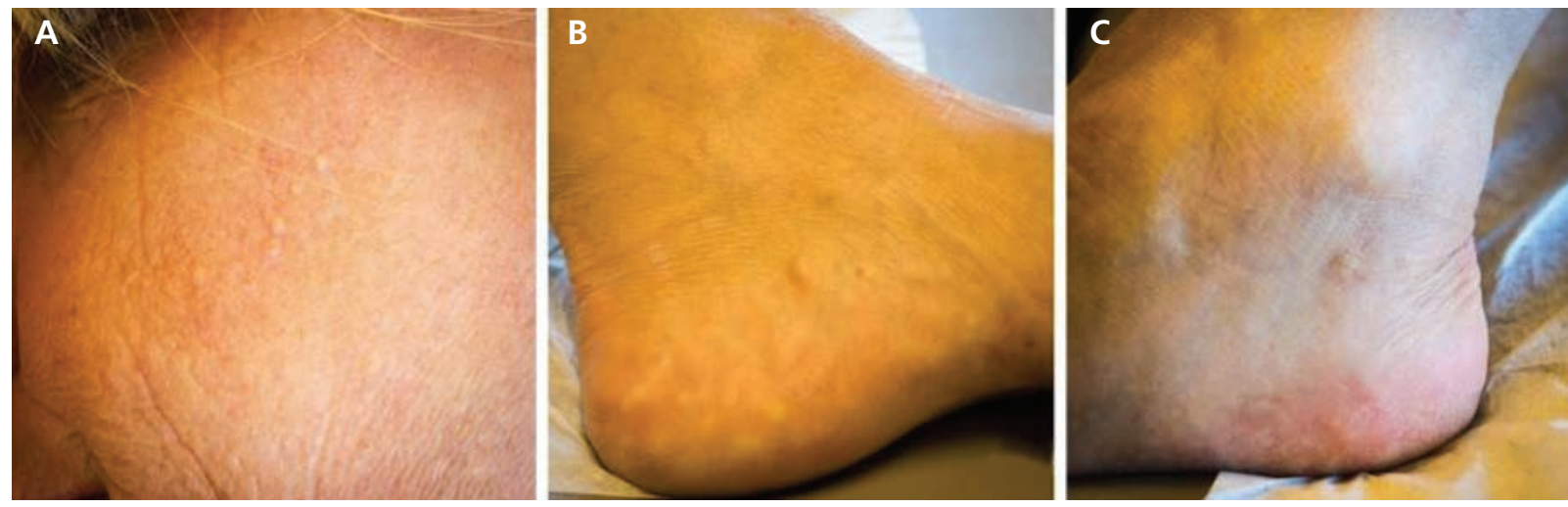

Figure 2 Pathognomonic mucocutaneous features of Cowden syndrome: (A) trichilemmomas and (B, C) acral keratoses.

and $35 \%$, respectively. ${ }^{7-10}$ Furthermore, patients with CS who have been diagnosed with one cancer have a 7-fold increased risk of developing subsequent cancers or second malignant neoplasms (SMNs). ${ }^{11}$ A recent multicenter prospective study of 2,912 patients with CS and CS-like syndromes revealed 114 patients (5.6\%) who had a documented PTEN mutation. Of these 114 patients, 46 (40\%) had SMNs; these were commonly breast, thyroid, endometrial, and renal cancers. Colorectal cancer and melanoma were also documented as SMNs, but there was no report of Ewing sarcoma. ${ }^{11}$ Previous reports documented the presentation of rare bony and soft tissue sarcomas in association with CS, such as osteosarcoma ${ }^{12}$ and liposarcoma, ${ }^{13}$ but no reports mentioned Ewing sarcoma. A search through PubMed using the terms "Cowden syndrome" and "Ewing sarcoma" could not identify any other cases.

Ewing sarcoma is a rare, small round blue cell tumor with characteristic translocations, thought to be of neural origin, that classically develops in the bones or soft tissues of children and adolescents, with an incidence peak at age 15 to 17 years. The present patient developed the disease at age 46 years, 17 years after her breast cancer diagnosis. The impact of age on outcome is controversial; limited data have indicated that the outcome for adults is not as promising as for adolescents, ${ }^{14,15}$ but other studies have shown that adults are not always treated with the same chemotherapy dose intensity as children. ${ }^{16,17}$

There is no clear molecular relationship between CS and Ewing sarcoma. CS is an autosomal dominant inherited disease with incomplete penetrance and variable expressivity, mostly in PTEN, a tumor suppressor gene located on chromosome 10q23.3.1,2
PTEN encodes for a 403-amino acid protein and acts as a lipid phosphatase that leads to hypoactivity of the PI3K/AKT/mTOR pathway. ${ }^{18,19}$ Loss or reduced PTEN activity results in increased phosphorylation of cellular proteins that induce cell cycle progression, altered metabolism, growth, migration, and invasion. ${ }^{20}$ Phenotypically, these mutations result in characteristic features, such as macrocephaly, intestinal hamartomatous polyps, benign skin lesions, and neoplastic transformation. Studies have shown that the deletion or reduction of PTEN can lead to breast cancer, ${ }^{21}$ melanoma, ${ }^{22}$ thyroid cancer, ${ }^{23}$ endometrial cancer, ${ }^{24}$ and other types of cancers. Small subsets of patients with CS or a CS-like syndrome may have mutations involving KLLN, SDHB, SDHC, and SDHD, and PIK3CA or AKT $1 .{ }^{14}$

Meanwhile, the Ewing sarcoma family of tumors are a group of malignant bone or soft tissue tumors most commonly characterized by the unique $\mathrm{t}(11 ; 22)(\mathrm{q} 24 ; \mathrm{q} 12)$ translocation, resulting in EWS-FLI1 fusion. ${ }^{25}$ In addition to the characteristic translocations, there are 2 sources of variability: the EWS fusion partner (eg, FLI1, ERG, ETV1, $E 1 A$, or $F E V$ ) and the breakpoint location within the genes. Somatic alterations in known tumor suppressor genes have been identified in Ewing sarcoma, including CDKN2A, ${ }^{26}$ TP53, ${ }^{27,28}$ and recently SOX6 and PTEN. ${ }^{29}$ The rate of PTEN anomalies in Ewing sarcoma was documented at $14.2 \% .^{29}$ Studies also showed that PTEN and TP53 regulate and enhance each other's tumor-suppressive function. ${ }^{30,31}$ Although PTEN loss has been evidenced in Ewing sarcoma, it does not appear to be a driver mutation, because Ewing sarcoma had not been noted in patients with CS until this report. 
Future treatment directions of CS and Ewing sarcoma may involve targeting PTEN loss using an mTOR inhibitor. Sirolimus (or rapamycin, an mTORC1 inhibitor) was found to decrease mucocutaneous lesions in a CS mouse model ${ }^{32}$ and improve skin lesions, gastrointestinal lesions, and cerebellar function of 16 patients with PHTs in a recent clinical trial. ${ }^{33}$ For Ewing sarcoma, preclinical data of mTOR inhibition yielded favorable results. A phase II trial using an mTOR inhibitor, ridaforolimus, in advanced soft tissue and bone sarcomas, including Ewing sarcoma, increased median progression-free survival (PFS) to 15.3 weeks and overall survival (OS) to 40 weeks. $^{34}$ A subsequent phase III trial showed improvement in PFS compared with placebo (median PFS, 17.7 vs 14.6 weeks, respectively) and a decrease in target size, but did not statistically improve OS (median OS, 90.6 vs 85.3 weeks, respectively). ${ }^{35}$

In the present patient, at the time of writing both her breast cancer and Ewing sarcoma have been treated successfully with conventional chemotherapy, radiation therapy, and surgery. She will be closely surveilled for the occurrence of new neoplasms in several organs. An mTOR inhibitor may be indicated if she develops symptomatic skin, gastrointestinal, or neurologic lesions in the future.

\section{Conclusions}

This case report is the first to describe the coexistence of Ewing sarcoma of the pelvis in a patient with CS. Surveillance for other secondary malignancies linked to PTEN mutation should be performed regularly and thoroughly in patients with PTEN-mutant CS to ensure a prompt diagnostic workup and proper management. The rare occurrence of synchronous CS and Ewing sarcoma in this patient also emphasizes the need for further research.

\section{Acknowledgments}

The authors would like to thank Charis Eng, MD, $\mathrm{PhD}$, of the Cleveland Clinic, who helped to diagnose this patient and for input regarding this manuscript.

\section{References}

1. Liaw D, Marsh DJ, Li J, et al. Germline mutations of the PTEN gene in Cowden disease, an inherited breast and thyroid cancer syndrome. Nat Genet 1997;16:64-67.

2. Nelen MR, van Staveren WC, Peeters EA, et al. Germline mutations in the PTEN/MMAC1 gene in patients with Cowden disease. Hum Mol Genet 1997;6:1383-1387.

3. Starink TM, Hausman R. The cutaneous pathology of extrafacial lesions in Cowden's disease. J Cutan Pathol 1984;11:338-344.

4. Starink TM, Hausman R. The cutaneous pathology of facial lesions in Cowden's disease. J Cutan Pathol 1984;11:331-337.

5. Daly MB, Axilbund JE, Buys S, et al. Genetic/familial high-risk assessment: breast and ovarian. J Natl Compr Canc Netw 2010;8:562-594. To view the most recent version of these guidelines, visit NCCN.org.

6. Farooq A, Walker LJ, Bowling J, Audisio RA. Cowden syndrome. Cancer Treat Rev 2010;36:577-583.

7. Bubien V, Bonnet F, Brouste V, et al. High cumulative risks of cancer in patients with PTEN hamartoma tumour syndrome. J Med Genet 2013;50:255-263.

8. Ngeow J, Mester J, Rybicki LA, et al. Incidence and clinical characteristics of thyroid cancer in prospective series of individuals with Cowden and Cowden-like syndrome characterized by germline PTEN, SDH, or KLLN alterations. J Clin Endocrinol Metab 2011;96:E2063-2071.

9. Nieuwenhuis $\mathrm{MH}$, Kets CM, Murphy-Ryan M, et al. Cancer risk and genotype-phenotype correlations in PTEN hamartoma tumor syndrome. Fam Cancer 2013;13:57-63.

10. Tan MH, Mester JL, Ngeow J, et al. Lifetime cancer risks in individuals with germline PTEN mutations. Clin Cancer Res 2012;18:400-407.

11. Ngeow J, Stanuch K, Mester JL, et al. Second malignant neoplasms in patients with cowden syndrome with underlying germline PTEN mutations. J Clin Oncol 2014;32:1818-1824.

12. Yen BC, Kahn H, Schiller AL, et al. Multiple hamartoma syndrome with osteosarcoma. Arch Pathol Lab Med 1993;117:1252-1254.

13. FaCD Online Syndrome Fact Sheet: Cowden Syndrome. Familial Cancer Database Web site. Available at: http://www.familialcancerdatabase.nl/ loggedin/syndromedetails.aspx?SyndromeCode=10. Accessed October 13, 2015.

14. Bacci G, Balladelli A, Forni C, et al. Adjuvant and neoadjuvant chemotherapy for Ewing sarcoma family tumors in patients aged between 40 and 60: report of 35 cases and comparison of results with 586 younger patients treated with the same protocols in the same years. Cancer 2007;109:780-786.

15. Baldini EH, Demetri GD, Fletcher CD, et al. Adults with Ewing's sarcoma/ primitive neuroectodermal tumor: adverse effect of older age and primary extraosseous disease on outcome. Ann Surg 1999;230:79-86.

16. Ahmed SK, Robinson SI, Okuno SH, et al. Adult ewing sarcoma: survival and local control outcomes in 102 patients with localized disease. Sarcoma 2013;2013:681425.

17. Kolb EA, Kushner BH, Gorlick R, et al. Long-term event-free survival after intensive chemotherapy for Ewing's family of tumors in children and young adults. J Clin Oncol 2003;21:3423-3430.

18. Cully M, You H, Levine AJ, Mak TW. Beyond PTEN mutations: the PI3K pathway as an integrator of multiple inputs during tumorigenesis. Nat Rev Cancer 2006;6:184-192.

19. Lee JO, Yang H, Georgescu MM, et al. Crystal structure of the PTEN tumor suppressor: implications for its phosphoinositide phosphatase activity and membrane association. Cell 1999;99:323-334.

20. Chu EC, Tarnawski AS. PTEN regulatory functions in tumor suppression and cell biology. Med Sci Monit 2004;10:RA235-241.

21. Li J, Yen C, Liaw D, et al. PTEN, a putative protein tyrosine phosphatase gene mutated in human brain, breast, and prostate cancer. Science 1997;275:1943-1947.

22. Wu H, Goel V, Haluska FG. PTEN signaling pathways in melanoma. Oncogene 2003;22:3113-3122.

23. Hou P, Liu D, Shan Y, et al. Genetic alterations and their relationship in the phosphatidylinositol 3-kinase/Akt pathway in thyroid cancer. Clin Cancer Res 2007;13:1161-1170.

24. Risinger JI, Hayes AK, Berchuck A, Barrett JC. PTEN/MMAC1 mutations in endometrial cancers. Cancer Res 1997;57:4736-4738.

25. Downing JR, Head DR, Parham DM, et al. Detection of the $(11 ; 22)$ (q24;q12) translocation of Ewing's sarcoma and peripheral neuroectodermal tumor by reverse transcription polymerase chain reaction. Am J Pathol 1993;143:1294-1300. 
Chandhanayingyong et al

26. Tsuchiya T, Sekine K, Hinohara S, et al. Analysis of the p16INK4, p14ARF, p15, TP53, and MDM2 genes and their prognostic implications in osteosarcoma and Ewing sarcoma. Cancer Genet Cytogenet 2000;120:9198.

27. Neilsen PM, Pishas KI, Callen DF, Thomas DM. Targeting the p53 pathway in Ewing sarcoma. Sarcoma 2010;2011:746939.

28. Radig K, Schneider-Stock R, Rose I, et al. p53 and ras mutations in Ewing's sarcoma. Pathol Res Pract 1998;194:157-162.

29. Lynn M, Wang Y, Slater J, et al. High-resolution genome-wide copynumber analyses identify localized copy-number alterations in Ewing sarcoma. Diagn Mol Pathol 2013;22:76-84.

30. Freeman DJ, Li AG, Wei G, et al. PTEN tumor suppressor regulates p53 protein levels and activity through phosphatase-dependent and -independent mechanisms. Cancer Cell 2003;3:117-130.
31. Stambolic V, MacPherson D, Sas D, et al. Regulation of PTEN transcription by $\mathrm{p} 53$. Mol Cell 2001;8:317-325.

32. Squarize CH, Castilho RM, Gutkind JS. Chemoprevention and treatment of experimental Cowden's disease by mTOR inhibition with rapamycin. Cancer Res 2008;68:7066-7072.

33. Komiya T, Blumenthal GM, Ballas MS, et al. A pilot study of sirolimus (S) in subjects with Cowden syndrome (CS) with germ-line mutations in PTEN [abstract]. J Clin Oncol 2013;31(Suppl):Abstract 2532.

34. Chawla SP, Staddon AP, Baker LH, et al. Phase II study of the mammalian target of rapamycin inhibitor ridaforolimus in patients with advanced bone and soft tissue sarcomas. J Clin Oncol 2012;30:78-84.

35. Demetri GD, Chawla SP, Ray-Coquard I, et al. Results of an international randomized phase III trial of the mammalian target of rapamycin inhibitor ridaforolimus versus placebo to control metastatic sarcomas in patients after benefit from prior chemotherapy. J Clin Oncol 2013;31:2485-2492. 